EPJ Web of Conferences 82, 01029 (2015)

DOI: $10.1051 /$ epjconf/20158201029

(C) Owned by the authors, published by EDP Sciences, 2015

\title{
A new approach to numerical analysis of reliability indices in electronics
}

\author{
Kuznetsov Geniy and Kravchenko Evgeny ${ }^{a}$ \\ Tomsk Polytechnic University, Lenina Av. 30, 634050 Tomsk, Russia
}

\begin{abstract}
Spatial modeling of unsteady temperature fields is conducted in a microelectronic printed circuit board (PCB) with an account of convective and radiation heat transfer with the environment. The data for numerical modeling of temperature fields serve as a basis for determining the aging characteristics of the polymer material as a structural component of electronic engineering products. The obtained results allow concluding on the necessity to consider spatial nonuniform temperature fields when estimating the degree of polymeric materials degradation at the continuous service of products, as well as on the impact of polymer aging on reliability features of microelectronic devices.
\end{abstract}

\section{Introduction}

The reliability of microelectronic products depends on numerous factors, including the materials used. Among a great variety of materials, such polymeric composites are widely spread as laminated plastic, dielectrics, plastics, compounds, and protecting polymeric coatings. These materials are indispensable for the implementation of any microelectronic device.

The properties of polymeric materials are subject to changes in the course of time. This is caused by complicated physical and chemical processes taking place under the combined action of internal and external factors during the operation of electronic devices [1]. The main factors affecting the degradation rate (aging) of polymers are the following: temperature [2], thermocycling [3], light and radiation impact [4], and ambient moisture [5]. In real operational conditions, two or more factors can affect the electronic equipment. For instance, the authors of the work described in [6] attempted to consider several factors in order to analyze degradation characteristics of polymeric materials.

It is known that electronic operates within a sufficiently narrow range of elevated working temperature, $40-800{ }^{\circ} \mathrm{C}$. However, the temperature increase even by $10 \%$ as a rule leads to the reduction of the reliability characteristics by $25-50 \%$ [7]. Hence, thermal influence is regarded [2, 3, 6, 7] as one of the crucial factors. At the same time, together with external thermal influences on microelectronic products, consideration should be given to the spatial nonuniformity of temperature fields in case of possible local overheating at functional assemblies of equipment [8]. Moreover, it should be noted that temperature gradients may also arise due to the degradation of thermal interface material during continuous operation [9] and under thermal cycling conditions [10] affecting microelectronic devices.

\footnotetext{
${ }^{a}$ Corresponding author: kevatp@tpu.ru
}

This is an Open Access article distributed under the terms of the Creative Commons Attribution License 4.0, which permits unrestricted use, distribution, and reproduction in any medium, provided the original work is properly cited. 
Table 1. Thermophysical characteristics of elements PCB.

\begin{tabular}{|c|c|c|c|}
\hline № area & Density $\rho, \mathrm{kg} / \mathrm{m}^{3}$ & $\begin{array}{c}\text { Specific heat } \mathrm{C}, \\
\mathrm{J} /(\mathrm{kg} \cdot \mathrm{K})\end{array}$ & $\begin{array}{c}\text { Thermal conductivity } \\
\lambda, \mathrm{W} /(\mathrm{m} \cdot \mathrm{K})\end{array}$ \\
\hline 1 & 2500 & 1088 & 2 \\
\hline 2 & 2700 & 900 & 210 \\
\hline 3 & 2900 & 1100 & 0,8 \\
\hline
\end{tabular}

Under the continuous thermal influence, the micro- and macro-structure of polymeric materials is changed, which is one of the possible reasons for the appearance of cracks and further destruction of the polymer $[3,4,6]$. Consequently, this can lead to the failure of a functional assembly and an electronic device as a whole. In view of the development trends of microelectronic industry, consisting in miniaturization of products with the increased heat release per unit of area and wide spread of polymeric materials, it becomes necessary to consider the polymer degradation at the reliability analysis.

Until now, the reliability characteristics have been estimated without regard for the destruction process of polymeric composite materials [11]. The studies consider the aging processes during the modeling of electronic engineering circuits, but pertaining to semiconducting elements of circuits only [11]. Moreover, no consideration is given to the spatial nonuniformity of temperature fields that arises both due to the difference in materials thermophysical characteristics and unsteadiness of thermal conditions occurring, for instance, at cyclic operation of equipment [3].

The objective of the paper is to determine the degradation numerical characteristics for such a quite widely used polymeric material as fiber-glass plastic (for example FR4) with account of spatial unsteadiness of temperature fields at the PCB of a typical design.

\section{Simulation methodology}

The degradation process of polymeric materials is temperature-dependent [12] and described by the equation:

$$
\frac{\mathrm{d} \eta}{\mathrm{dt}}=(1-\eta) \cdot \mathrm{k} \cdot \exp \left(-\frac{\mathrm{E}}{\mathrm{R} \cdot \mathrm{T}}\right) .
$$

At the initial time $\mathrm{t}=0, \eta=0$.

Where $\eta$-component aging, which characterizes the degree of degradation the polymer material, $\mathrm{t}$ - time, $\mathrm{k}$ - exponential factor, $\mathrm{E}$ - is the activation energy, $\mathrm{R}$-molar gas constant, $\mathrm{T}$ - the temperature.

Numerical modeling of aging characteristics of a polymeric material was performed at the example of the assembly which is typical for the modern electronic engineering and consists of a PCB and six local heat sources. The modeled object was a fiber-glass plastic board sized on axes $\mathrm{X}, \mathrm{Y}$ and $\mathrm{Z}$ in proportion to Lx, Ly, and Lz. The models of heat sources were represented by silicon transistors used in power amplifiers and switching devices, as well as by an integrated circuit as the most typical elements applied in microelectronic products. It was assumed that the conditions of equal thermal flows and temperatures were maintained at the boundaries with different thermophysical characteristics. The thermophysical characteristics of the materials applied to the elements of the printed assembly are tabulated.

Boundary conditions of type 3 with account of radiation were set at the interface with the environment.

In such context, the task comes to the solution of non-stationary thermal conductivity equation:

$$
C(x, y, z) \rho(x, y, z) \frac{\partial T}{\partial t}=\frac{\partial}{\partial x}\left(\lambda(x, y, z) \frac{\partial T}{\partial x}\right)+\frac{\partial}{\partial y}\left(\lambda(x, y, z) \frac{\partial T}{\partial y}\right)+\frac{\partial}{\partial z}\left(\lambda(x, y, z) \frac{\partial T}{\partial z}\right)+Q(t, x, y, z) .
$$




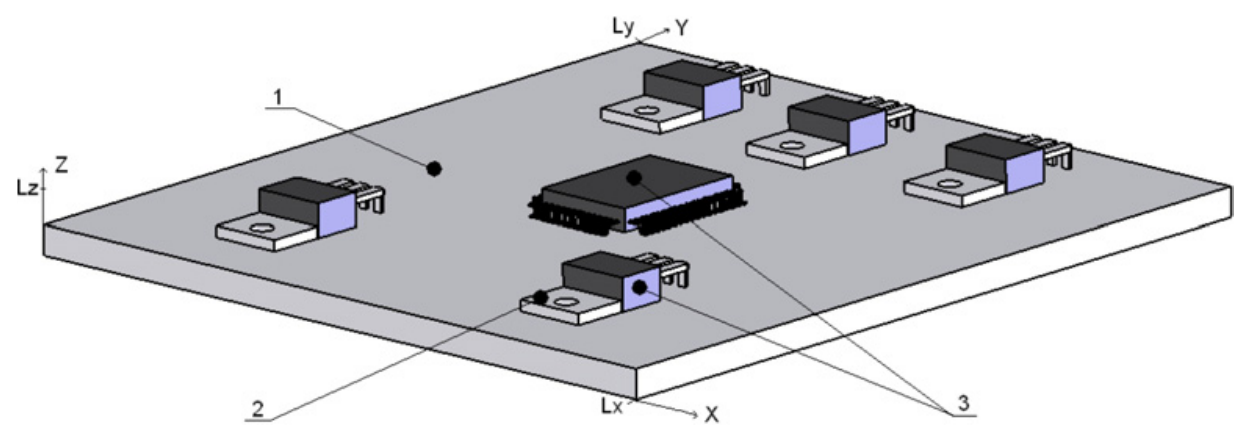

Figure 1. Geometry field solutions: 1 - circuit board; 2 - heat sink surface, 3 - case transistor and circuits.

Figure 1 shows a typical design of a PCB in electronic engineering.

For the purpose of comparison, two possible thermal conditions were examined. The first (real) one implies that the temperature at every point of the decision area is changed in accordance with the operating conditions of heat-generating elements within microelectronic devices and heat removal into the environment. The second one provides for assuming the temperature at every point of the decision area as constant and equal to $300 \mathrm{~K}$.

\section{Method of numerical solution}

The thermal conductivity Eq. (2) with appropriate initial and boundary conditions is solved by the finite difference method [13-15]. The diagram of splitting by coordinates was applied for the solution of difference analogues of a three-dimensional equation.

\section{Results of numerical investigations and discussion}

Numerical investigation of the temperature-dependence of the degradation factor $\eta$ characterizing the aging degree of fiber-glass plastic, a composite polymeric material, was conducted within the typical variation range of external influence parameters and PCB operational conditions. Spatial temperature fields were modeled at the computational mesh sized $110 \times 110 \times 18$ for the electronic PCB continuous operation $(\mathrm{t}=1.56 \cdot 108 \mathrm{sec})$. Heat generation power amounted to $1 \mathrm{~W}$ for the transistors, and $1 \mathrm{~W}$ for the microcircuit. Figure 2 shows a typical temperature field of a modeled PCB at the moment of time $(\mathrm{t}=1800 \mathrm{sec})$ in the cross-section of $\mathrm{Z}=0 \mathrm{~mm}$.

It should be noted that the temperature field presented in Fig. 2 is typical for the examined ranges and environment influences. Gradients $\mathrm{T}$ on axis $\mathrm{Z}$ reach 10 and more degrees. Besides, in the course of numerical investigation it was established that the operational mode of the modeled microelectronics product is unsteady to a significant extent.

At the same time, it should be noted that the most extreme thermal conditions were not considered at the modeling (the power of heat-generating sources being not more than $1 \mathrm{~W}$ ). It can also be supposed that the differences will be more significant with the growth of thermophysical characteristics nonuniformity on axis $\mathrm{Z}$.

Figure 3 shows the results of numerical modeling of the fiber-glass plastic aging degree in terms of operating time for the unsteady operational conditions of the modeled assembly of electronic equipment (curve 1) and for a certain constant (steady in terms of temperature) conditions (curve 2). In equation (1) the maximum temperature of the modeled assembly was chosen as a main factor influencing 


\section{EPJ Web of Conferences}

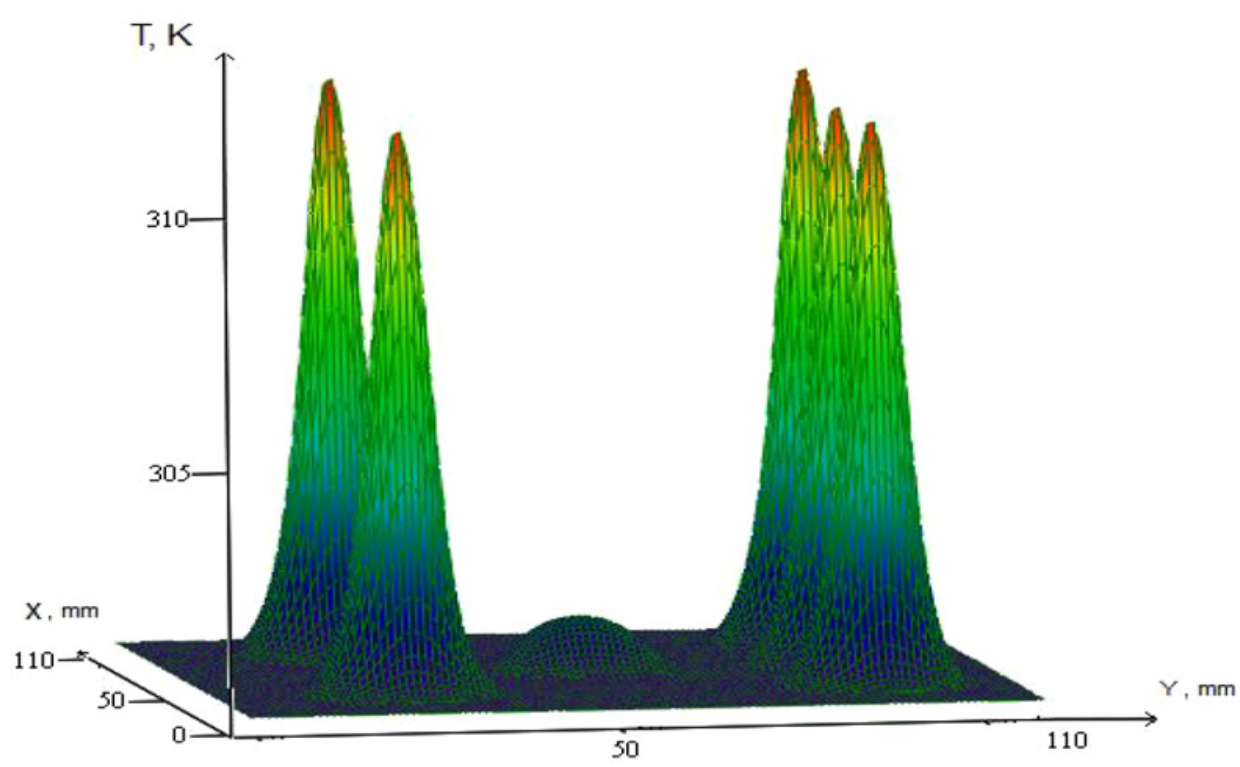

Figure 2. Temperature field simulated $\mathrm{PCB}$ (in cross-section $\mathrm{Z}=0 \mathrm{~mm}$ ).

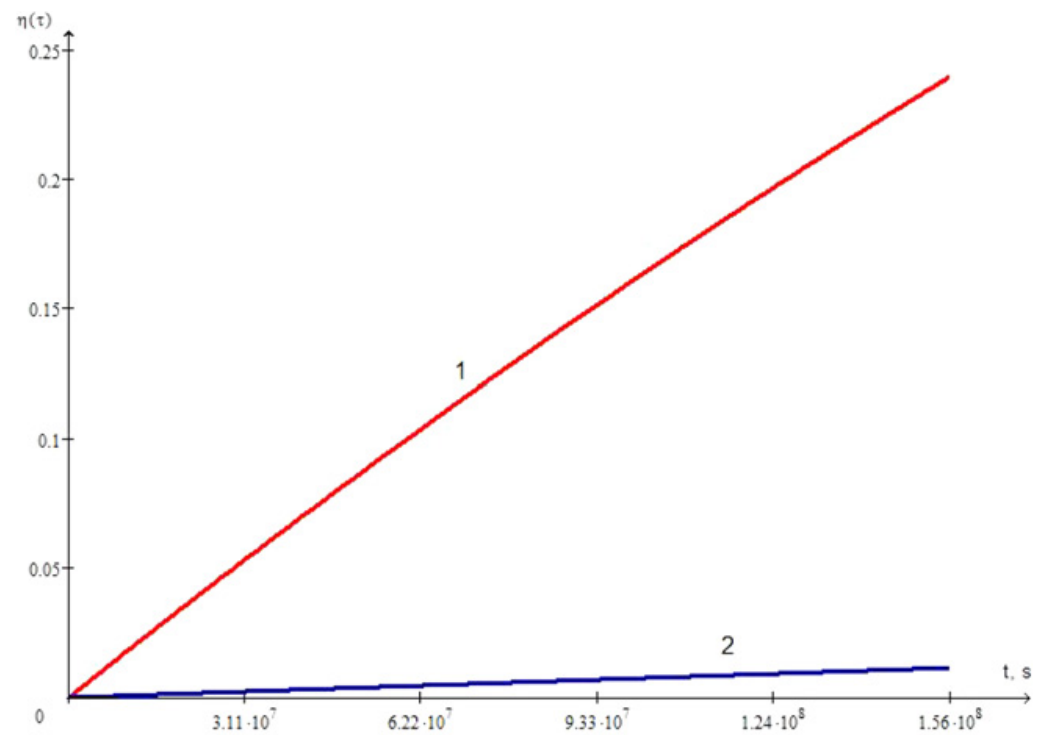

Figure 3. Dependence of the aging of polymeric materials from time to time. For nonstationary (1) and stationary (2) thermal mode $(\mathrm{T}=300 \mathrm{~K})$.

the polymeric material aging rate because the degradation process has an exponential temperaturedependent.

It was established that given the consideration of spatial nonuniformity of temperature field and unsteady operational thermal conditions, the aging factor $\eta$ is 20 times more than that for the stationary conditions in the examined electronic engineering product for a five-year operational period. Hence, the use of constant temperature at the estimation of polymeric materials aging degree is reasonable only for 
small durations and small power of heat generated by electronic device elements. It can also be noted that the values of thermochemical conversion level $\eta$ (aging degree) obtained in the course of numerical investigation for a polymeric composite material used in radio electronics describe such condition of the composite in five years of the PCB operation $(\eta=0.25)$ that cannot be regarded as satisfactory. The value $\eta=0.25$ corresponds to the physical state of breakage of a significant part of polymeric chain links and appropriate change, for example, of the composite dielectric and thermophysical characteristics.

It should be noted that numerical analysis of the investigated processes was conducted with the use of kinetic characteristics of fiber-glass plastic destruction that were determined from the solution of inverse problem of thermal aging for typical polymeric composite materials with the application of the device. At the same time, the examined conditions of the materials thermal destruction were not the most intense. It is known that the aging intensity of polymeric components used in the production of microelectronic devices is also highly dependent on the environment moisture content. The growth of moisture content has in some cases even higher impact than the temperature increase on a comparable scale. In view of this, the conclusion can be made that the results obtained within this study are most likely to be the basis for the lower bound of degradation degree of polymeric materials used in electronics.

\section{Conclusion}

The obtained results allow concluding that reliability characteristics should be analyzed with the consideration of real (spatial) temperature fields at microelectronic functional assemblies and units.

The consideration of aging characteristics of polymeric materials widely used in radio engineering devices for various applications and nonuniform unsteady temperature fields at specific functional assemblies will inevitably lead to a more accurate determination of reliability factors of the examined electronic technical devices and products. At the same time, the choice of one or another dependence of temperature time variation in the problems on determining the polymers destruction rate must be accompanied with the analysis of real heat release processes at electronic technical devices and products and heat transfer conditions both within the electronic devices and with the environment.

It is also important that the obtained regularities in the degradation mechanism of the construction materials applied in electronic engineering can be used for estimating the technical state in terms of the physical reliability theory. Such approach based on physic of failure (POF) was applied by the authors of the studies described in [11]. It consists in the fact that POF methods are essential component of the PDfR concept (probabilistic design for reliability) ensuring more efficient control of the cost for the development of electronic technical devices and the period required to introduce a product in the market.

The reported study was supported by the Grant of the Russian Scientific Fund (project №. 14-39-00003).

\section{References}

[1] H.J.L. Bressers, W.D. van Driel, K.M.B. Jansen, L.J. Ernst, G.Q. Zhang, Microelectronics Reliability, 47 (2007)

[2] R. Chaware, N. Vichare, P. Borgesen, D. Blass, K. Srihari, Proceedings of 2004 Surface Mount Technology Association International (2004)

[3] R.L. Torrisi, V. Maiorana, R. Nicolosi, G. Presti, Microelectronics Reliability, 52 (2012)

[4] V.K. Milinchuk, O.F. Pasevich, Je.R. Klinshpont, I.P. Sheluhov, T.N. Smirnova, High Energy Chemistry, 38 (2004)

[5] O. Thomas, C. Hunt, M. Wickham, Microelectronics Reliability, 52 (2012)

[6] B.C. Ray, Journal of Colloid and Interface Science, 298 (2006) 
[7] T.T. Mattila, Jue Li, JK Kivilahti, Microelectronics Reliability, 52 (2012)

[8] K.N. Tu, Microelectronics Reliability, 51 (2011)

[9] R. Skuriat, J.F. Li, P.A. Agyakwa, N. Mattey, P. Evans, C.M. Johnson, Microelectronics Reliability, 53 (2013)

[10] J.P. Ousten, Z. Khatir, Microelectronics Reliability, 51 (2011)

[11] S. Ephraim, Microelectronics Reliability, 53 (2013)

[12] S.N. Zhurkov, Int J Fract Mech., 1 (4), (1965)

[13] G.V. Kuznetsov, E.V. Kravchenko, Elektromagnitnye Volny i Elektronnye Systemy, 10 (11-12), (2005)

[14] G.V. Kuznetsov, E.V. Kravchenko, Journal of Engineering Physics and Thermophysics, 80 (5), (2007)

[15] E.V. Kravchenko, G.V. Kuznetsov, EPJ Web of Conferences, 76 (2014) 